International Journal of Pure and Applied Mathematics

Volume 90 No. 1 2014, 57-64

ISSN: 1311-8080 (printed version); ISSN: 1314-3395 (on-line version)

url: http://www.ijpam.eu

doi: http://dx.doi.org/10.12732/ijpam.v90i1.7

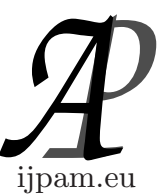

\title{
ON DECOMPOSITIONS OF ORDERED SEMIGROUPS
}

\author{
Thawhat Changphasr \\ Department of Mathematics \\ Faculty of Science \\ Khon Kaen University \\ Khon Kaen 40002, THAILAND
}

\begin{abstract}
The purpose of this paper is to introduce and describe $a$-connected ordered semigroups and weakly extremely commutative ordered semigroups. The results obtained generalize the results on semigroups without order.
\end{abstract}

AMS Subject Classification: $06 \mathrm{~F} 05$

Key Words: semigroup, ordered semigroup, external commutative, weakly extremely commutative, $a$-connected, semilattice, congruence, semilattice congruence

\section{Preliminaries}

Decompositions of semigroups without order has been studied by Protič and Stevanovič in [3]. The authors introduced and studied $a$-connected semigroups and weakly extremely commutative semigroups. The main result is as follows: a weakly extremely commutative semigroup is a semilattice of $a$-connected semigroups. This paper deals with ordered semigroups, the results obtained extended the result mentioned before.

We now recall some definitions and results used throughout this paper.

Received: September 25, 2013

(c) 2014 Academic Publications, Ltd. url: www.acadpubl.eu 
A semigroup $(S, \cdot)$ together with a partial order $\leq$ that is compatible with the semigroup operation, meaning that, for $x, y, z \in S$,

$$
x \leq y \Rightarrow z x \leq z y, x z \leq y z
$$

is called an ordered semigroup $([1],[2])$. In this paper, we write $(S, \cdot, \leq)$ for an ordered semigroup $(S, \cdot)$ with a partial order $\leq$ and write $x y(x, y \in S)$ for $x \cdot y$.

An element 0 of an ordered semigroup $(S, \cdot, \leq)$ is called a zero element $[1]$ if $0 x=x 0=0$ and $0 \leq x$ for all $x \in S$.

A mapping $\varphi: S \rightarrow T$ from an ordered semigroup $\left(S, \cdot, \leq_{S}\right)$ into an ordered semigroup $\left(T, \cdot, \leq_{T}\right)$ is called a homomorphism [6] if, for all $x, y \in S$,

(i) $\varphi(x y)=\varphi(x) \varphi(y)$;

(ii) $x \leq_{S} y \Rightarrow \varphi(x) \leq_{T} \varphi(y)$.

If $A$ and $B$ are nonempty subsets of an ordered semigroup $(S, \cdot, \leq)$, we write subsets $A B$ and $(A]$ of $S$ as follows:

$$
\begin{gathered}
A B=\{x y \mid x \in A, y \in B\} \\
(A]=\{x \in S \mid x \leq a \text { for some } a \in A\} .
\end{gathered}
$$

For $x \in S$, we write $A x$ and $x A$ for $A\{x\}$ and $\{x\} A$, respectively.

In [5], the following statements hold for any nonempty subsets $A, B$ of an ordered semigroup $(S, \cdot, \leq)$ :

(1) $A \subseteq(A]$;

(2) $A \subseteq B \Rightarrow(A] \subseteq(B]$;

(3) $(A](B] \subseteq(A B]$.

Definition 1. ([4]) Let $(S, \cdot, \leq)$ be an ordered semigroup. An equivalence relation $\rho$ on $S$ is called a congruence on $S$ if, for $x, y, z \in S$, if $x \rho y$ then $z x \rho z y$ and $x z \rho y z$.

Definition 2. ([4]) Let $(S, \cdot, \leq)$ be an ordered semigroup. A congruence $\rho$ on $S$ is called a semilattice congruence if, for $x, y \in S$,

(i) $x \rho x^{2}$;

(ii) $x y \rho y x$. 


\section{Decompositions of Ordered Semigroups}

We begin with the concept of $a$-connected as follows:

Definition 3. Let $(S, \cdot, \leq)$ be an ordered semigroup and let $a \in S$. Then two elements $x, y \in S$ are said to be a-connected if

$$
(x a)^{n} \in(y a S] \text { and }(y a)^{m} \in(x a S]
$$

for some positive integers $m, n$. If every element of $S$ is $a$-connected then $S$ is said to be an a-connected ordered semigroup.

Note from the definition above that if $(x a)^{n} \in(y a S]$ and $(y a)^{m} \in(x a S]$ for some positive integers $m$ and $n$, then

$$
(x a)^{p} \in(y a S] \text { and }(y a)^{p} \in(x a S]
$$

where $p=\max \{m, n\}$.

Definition 4. An ordered semigroup $(S, \cdot, \leq)$ is called an extremely commutative ordered semigroup if

$$
x y z=z y x
$$

for all $x, y, z \in S$.

Definition 5. An ordered semigroup $(S, \cdot, \leq)$ is called a weakly extremely commutative ordered semigroup if there exists $a \in S$ such that

$$
x a y=y a x
$$

for all $x, y \in S$.

It is clear that every extremely commutative ordered semigroup is a weakly extremely commutative ordered semigroup.

Example 6. Let $\left(K, \cdot \leq_{K}\right)$ be a commutative ordered semigroup and let $\left(T, \cdot \leq_{T}\right)$ is an ordered semigroup with zero. Let $\varphi: T \backslash\{0\} \rightarrow K$ be a homomorphism. Let $S=K \cup T \backslash\{0\}$. Define a multiplication on $S$, denoted by $\circ$, as follows:

(1) For $A, B \in T$,

$$
A \circ B=A B \text { if } A B \in T \backslash\{0\} ; A \circ B=\varphi(A) \varphi(B) \text { if } A B=0 ;
$$

(2) For $c, d \in K, c \circ d=c d$; 
(3) For $A \in T, c \in K, A \circ c=\varphi(A) c, c \circ A=c \varphi(A)$.

Define a partial order on $S$, denoted by $\leq_{S}$, as follows:

(4) For $A, B \in T, A \leq_{S} B \Leftrightarrow A \leq_{T} B$;

(5) For $c, d \in K, c \leq_{S} d \Leftrightarrow c \leq_{K} d$;

(6) For $A \in T, c \in K$,

$$
A \leq_{S} c \Leftrightarrow \varphi(A) \leq_{K} c \text { and } c \leq_{S} A \Leftrightarrow c \leq_{K} \varphi(A) .
$$

It is a routine matter to verify that $\left(S, \circ, \leq_{S}\right)$ is an ordered semigroup. If $A, B \in T \backslash\{0\}$ and $s \in K$, then

$$
\begin{aligned}
A \circ s \circ B=(\varphi(A) s) \circ B & =(\varphi(A) s) \varphi(B) \\
& =\varphi(A) s \varphi(B) \\
& =\varphi(B) s \varphi(A) \\
& =(B \circ s) \varphi(A) \\
& =B \circ s \circ A
\end{aligned}
$$

Hence $S$ is a weakly extremely commutative ordered semigroup.

The following two lemmas can be proved in the same manner as the proofs of Lemma 1 and Lemma 2 in [3].

Lemma 7. If $(S, \cdot, \leq)$ is a weakly extremely commutative ordered semigroup and if

$$
B=\{a \in S \mid \forall x, y \in S, x a y=y a x\}
$$

then $B$ is nonempty and $S B S \subseteq B$.

Proof. By assumption we have $B$ is nonempty. Let $a \in B$ and $u, v \in S$. To show that $u a v \in B$, we let $x, y \in S$. Since $x u, v y \in S$, it follows that

$$
x(u a v) y=(x u) a(v y)=(v y) a(x u)=v(x a y) u=y(u a v) x,
$$

and hence $u a v \in B$.

Lemma 8. Let $(S, \cdot, \leq)$ be a weakly extremely commutative ordered semigroup, $a \in B$ and $x, y \in S$. Then

$$
(x a y)^{2 k}=(x a)^{2 k-1} y^{2 k-1} x a y \text { and }(x a y)^{2 k+1}=(x a)^{2 k+1} y^{2 k+1}
$$


for all positive integers $k$.

Proof. We shall prove the assertion by induction. By Lemma 7, xay $\in B$. If $k=1$, then

$$
(x a y)^{2}=(x a y)(x a y)
$$

and

$$
(x a y)^{3}=x a y(x a y) x a y=x a x a(x a y) y y=(x a)^{3} y^{3} .
$$

We have

$$
(x a y)^{2 k+2}=(x a y)^{2 k+1}(x a y)=(x a)^{2 k+1} y^{2 k+1}(x a y)
$$

and

$$
\begin{aligned}
(x a y)^{2 k+3} & =(x a y)^{2 k+2}(x a y)=(x a)^{2 k+1} y^{2 k+1}(x a y) x a y \\
& =(x a)^{2 k+1} x a(x a y) y^{2 k+1} y=(x a)^{2 k+3} y^{2 k+3} .
\end{aligned}
$$

Therefore, the assertion holds.

Corollary 9. Let $(S, \cdot, \leq)$ be a weakly extremely commutative ordered semigroup, $a \in B$ and $x, y \in S$. Then

$$
(x a y)^{m} \in(x a)^{m-1} S
$$

for all positive integers $m$.

Proof. This is a consequence of Lemma 8.

We now prove the main theorem of this paper:

Theorem 10. Let $(S, \cdot, \leq)$ be a weakly extremely commutative ordered semigroup and let $a \in B$. Then $S$ is a semilattice of a-connected ordered semigroups.

Proof. We define a relation $\rho$ on $S$ by, for $x, y \in S$,

$$
x \rho y
$$

if and only if

$$
(x a)^{n} \in(y a S] \text { and }(y a)^{n} \in(x a S] \text { for some positive integer } n .
$$

To prove the theorem, it suffices to show that $\rho$ is a semilattice congruence on $S$.

If $x \in S$, then $(x a)^{2} \in(x a S]$, and so $x \rho x$. It is clear that, for $x, y \in S$, if $x \rho y$ then $y \rho x$. Let $x, y, z \in S$ be such that $x \rho y$ and $y \rho z$; thus 


$$
(x a)^{n} \in(y a S],(y a)^{n} \in(x a S] \text { and }(y a)^{m} \in(z a S],(z a)^{n} \in(y a S]
$$

for some positive integers $m, n$. Let $s, s^{\prime} \in S$ such that

$$
(x a)^{n} \leq \text { yas and }(z a)^{m} \leq y^{\prime} s^{\prime} .
$$

By Corollary 9, we obtain

$$
\begin{aligned}
(x a)^{(n+1)(m+1)} & =(x a)^{n(m+1)}(x a)^{m+1} \\
& \leq(y a s)^{m+1}(x a)^{m+1} \\
& \in(y a)^{m} S(x a)^{m+1} \\
& \subseteq(z a S]
\end{aligned}
$$

and

$$
\begin{aligned}
(z a)^{(n+1)(m+1)} & =(z a)^{m(n+1)}(z a)^{n+1} \\
& \leq\left(y a s^{\prime}\right)^{n+1}(z a)^{n+1} \\
& \in(y a)^{n} S(z a)^{n+1} \\
& \subseteq(x a S] .
\end{aligned}
$$

Thus $x \rho z$. Therefore, $\rho$ is an equivalent relation on $S$.

To show that $\rho$ is a congruence on $S$, let $x, y, z \in S$ be such that $x \rho y$. Then

$$
(x a)^{n} \in(y a S],(y a)^{n} \in(x a S]
$$

for some positive integer $n$; thus $(x a)^{2 n+1} \in(y a S]$. Since $a z \in B$, it follows that

$$
\begin{aligned}
(x z a)^{2 n+2} & =x(z a x)^{2 n+1} z a=x(x a z)^{2 n+1} z a \\
& =x(x a)^{2 n+1} z^{2 n+1} z a=x(x a)^{2 n+1} z z^{2 n+1} a \\
& \in x(y a S] z z^{2 n+1} a \subseteq(x y a S z] z^{2 n+1} a \\
& \subseteq(x z a S y] z^{2 n+1} a \subseteq(y z a S x] z^{2 n+1} a \\
& \subseteq(y z a S]
\end{aligned}
$$

Similarly, $(z y a)^{2 n+2} \in(x z a S]$, and so $x z \rho y z$. We have

$$
\begin{aligned}
(z x a)^{2 n+2} & =z(x a z)^{2 n+1} x a=z(x a)^{2 n+1} z^{2 n+1} x a \\
& \in(z y a S] z^{2 n+1} x a \subseteq(z y a S]
\end{aligned}
$$

and, similarly, $(z y a)^{2 n+2} \in(z x a S]$. Hence $\rho$ is a congruence on $S$.

Finally, we shall show that $\rho$ is a semilattice congruence. Let $x, y \in S$. Since $x a, x^{2} a \in B$, so 


$$
\left(x^{2} a\right)^{3}=x x\left(a x^{2}\right) a x^{2} a=x a\left(a x^{2}\right) x x^{2} a \in a x S
$$

and

$$
(x a)^{3}=x a x a x a=x x a(x a) a \in x^{2} a S
$$

That is $x \rho x^{2}$. We have

$$
(x y a)^{2}=x(y a x) y a=y(y a x) x a=y y(a x) x a=y x a(a x) y \in y x a S .
$$

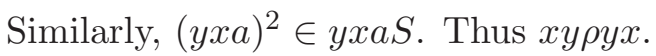

Corollary 11. An extremely commutative ordered $\operatorname{semigroup}(S, \cdot, \leq)$ is a semilattice of $a$-connected ordered semigroups for every $a \in B$.

Proof. Obvious.

Definition 12. An ordered semigroup $(S, \cdot, \leq)$ is said to be medial ordered semigroup if

$$
x y z t=x z y t
$$

for all $x, z, y, t \in S$.

Theorem 13. A medial ordered semigroup $(S, \cdot, \leq)$ is a band of aconnected ordered semigroups for every $a \in S$, that is there exists a congruence $\rho$ on $S$ such that $x \rho x^{2}$ for all $x \in S$ and each $\rho$-class is an a-connected ordered semigroup.

Proof. Let $a \in S$. Define a relation $\rho$ on $S$ by $x \rho y$ if and only if $(x a)^{n} \in(y a S]$ and $(y a)^{n} \in(x a S]$.

for some positive integer $n$. As in the Proof of Theorem 10, $\rho$ is an equivalence relation.

To show that $\rho$ is a congruence on $S$, we let $x, y, z \in S$ be such that $x \rho y$. Then

$$
(x a)^{n} \in(y a S] \text { and }(y a)^{n} \in(x a S] .
$$

for some positive integer $n$. Since $S$ is a medial ordered semigroup, we have

$$
(x z a)^{n+1}=(x a)^{n} z^{n+1} x a \in(y a S] z z^{n} x a \subseteq(y z a S] z^{n} x a \subseteq(y z a S] .
$$

Similarly, $(y z a)^{n+1} \in(x z a S]$. In the same manner, we have

$$
(z x a)^{n+1} \in(z y a S] \text { and }(z y a)^{n+1} \in(z x a S] .
$$


Thus $\rho$ is a congruence on $S$.

To show that $x \rho x^{2}$ for all $x \in S$, we let $x \in S$. Then

$$
\left(x^{2} a\right)^{2}=x^{2} a x^{2} a=x a x^{3} a \in x a S \subseteq(x a S]
$$

and

$$
(x a)^{2}=x a x a=x^{2} a a \in x^{2} a S \subseteq\left(x^{2} a S\right],
$$

and thus $x \rho x^{2}$. It is clear by the definition of $\rho$ that each $\rho$-class is an $a$ connected.

\section{References}

[1] G. Birkhoff, Lattice Theory (Vol. XXV), Rhode Island, Amer. Math. Soc., Coll. Pub., 1967.

[2] L. Fuchs, Partially Ordered Algebraic Systems, Great Britain: AddisonWesley Publ. Comp., 1963.

[3] P.V. Protič, N. Stevanovič, Some decompositions of semigroups, Matematiqki Vesnik, 61 (2009), 153-158.

[4] N. Kehayopulu, Remark on ordered semigroups, Mathematica Japonica, 6, No. 35 (1990), 1061-1063.

[5] N. Kehayopulu, M. Tsingelis, On left regular ordered semigroups, Southeast Asian Bulletin of Mathematics, 25 (2002), 609-615.

[6] N. Kehayopulu, M. Tsingelis, On subdirectly irreducible ordered semigroups, Semigroup Forum, 50 (1995), 161-177. 